\title{
Equilibrium and nonequilibrium gap-state distribution in amorphous silicon
}

\author{
J. M. Asensi and J. Andreu \\ Departament de Física Aplicada i Electrònica, Universitat de Barcelona, Diagonal 647, E-08028 Barcelona, Spain
}

(Received 17 November 1992; revised manuscript received 10 February 1993)

\begin{abstract}
A general and straightforward analytical expression for the defect-state-energy distribution of $a$-Si:H is obtained through a statistical-mechanical treatment of the hydrogen occupation for different sites. Broadening of available defect energy levels (defect pool) and their charge state, both in electronic equilibrium and nonequilibrium steady-state situations, are considered. The model gives quantitative results that reproduce different defect phenomena, such as the thermally activated spin density, the gap-state dependence on the Fermi level, and the intensity and temperature dependence of light-induced spin density. An interpretation of the Staebler-Wronski effect is proposed, based on the "conversion" of shallow charged centers to neutrals near the middle of the gap as a consequence of hydrogen redistribution.
\end{abstract}

\section{INTRODUCTION}

Considerations of chemical equilibrium in $a-\mathrm{Si}: \mathrm{H}$ are used to test microscopic models of defect formation. The key idea for the chemical approach is that defects can be thought of as the product of solid-state reactions in thermodynamical equilibrium above a freeze-in temperature $T^{*} .^{1-10}$ In particular, hydrogen-mediated SiSi bondbreaking reactions have been proposed as one of the more reasonable microscopic mechanisms of defect formation. Two approaches have been considered, which can be represented by the following reactions: ${ }^{6}$

$$
\mathrm{SiH}+\mathrm{SiSi} \leftrightarrows D+\mathrm{SiHSi}
$$

In reaction (1) a spatially isolated $\mathrm{SiH}$ bond interacts with a weak SiSi bond

$$
2 \mathrm{SiH}+\mathrm{SiSi} \leftrightarrows 2 D+\mathrm{SiHSiH},
$$

producing an isolated dangling bond $(D)$ and an intimate dangling-bond-bonded hydrogen pair (SiHSi). In reaction (2), it is assumed that the SiHSi center is finally saturated by another hydrogen atom.

The incorporation of the statistical broadening of available defect energy levels, i.e., the defect pool concept, and the dependence of the formation enthalpies of (1) and (2) on the Fermi energy permit quantitative descriptions of the distribution of defect states in the gap.

However, while the chemical equilibrium model with the defect pool concept satisfactorily describes defect creation by doping and thermal quenching, ${ }^{1}$ and can account for the apparent shift in defect levels with doping, ${ }^{3,4}$ quantitative analysis of other defect processes is less obvious. We refer in particular to the light-induced defect generation and the correlation between the amount of hydrogen evolved from a specimen with deep level density.

Limitations to the description based on reaction (1) have been suggested. The difficulties arise from the requirement of exact equality of the densities of two different kinds of defect ( $D$ and SiHSi). ${ }^{7}$ Nevertheless, experimental results and theoretical calculations of hydro- gen bonding configurations appear to be most easily reconciled with a model based on reaction (2). In particular, Zafar and Schiff ${ }^{8}$ have proposed a microstructural description of this reaction based on two known phases (dilute and clustered) of bonded hydrogen in $a$-Si:H. ${ }^{11}$ The analysis of their model in terms of partition functions and hydrogen chemical potential facilitates a general and straightforward approach to chemical equilibrium based on hydrogen-mediated reactions. So, a simple estimation of the hydrogen binding energies for the different hydrogen sites in reaction (2) can account for the thermally activated paramagnetic defect density and the changes in spin density observed following hydrogen evolution.

In this paper we present an extension of the statistical analysis of reaction (2), which incorporates the defect pool concept and the dependence of the defect formation energies on the Fermi level. From this treatment a general solution for the gap-state distribution in $a-\mathrm{Si}: \mathrm{H}$ is deduced. We will demonstrate that this solution is valid to describe the steady-state defect density under nonequilibrium conditions and can account quantitatively for the dependence of the metastable defect density in $a-\mathrm{Si}: \mathrm{H}$ on temperature and light intensity. On the other hand, explicit use of hydrogen chemical potential allows a more realistic description of complex situations, such as hydrogen-depleted $a-\mathrm{Si}: \mathrm{H}$, defect profiles due to Fermilevel displacement, and $a-\mathrm{Si}: \mathrm{H}$ growth under visible-light illumination. The statistical approach also allows us to reconsider some of the hypotheses implicit in the more conventional treatment, based on the application of the law of mass action.

\section{THEORY}

\section{A. Equilibrium gap-state distribution}

Equilibrium reaction (2) includes the involvement of two hydrogen binding sites: dangling-bond sites $(D)$, which can bind only a single hydrogen atom, and weakbond sites ( $\mathrm{SiSi}$ ), which can bind only two hydrogen 
atoms. Assuming that the underlying total densities $D_{T}$ and $\mathrm{SiSi}_{T}$ do not vary, the equilibrium densities of the various hydrogenation states can be obtained from the partition functions describing the two centers with a common temperature $T^{*}$, and hydrogen chemical potential $\mu_{\mathrm{H}}$.
In what follows we consider the isolated dangling-bond site. It can be either completely unoccupied $(D)$ or singly occupied $(\mathrm{SiH})$. The unoccupied state $D$ can present three possible charge states $D^{0}, D^{+}$, and $D^{-}$, with probabilities $f^{0}, f^{+}$, and $f^{-}$when electronic equilibrium is assumed: ${ }^{12}$

$$
\begin{aligned}
& f^{0}(E)=\frac{2 \exp \left[-\left(E-E_{F}\right) / k T^{*}\right]}{1+2 \exp \left[-\left(E-E_{F}\right) / k T^{*}\right]+\exp \left[-\left(2 E+U_{\mathrm{eff}}-2 E_{F}\right) / k T^{*}\right]}, \\
& f^{+}(E)=\frac{1}{2} \exp \left[\left(E-E_{F}\right) / k T^{*}\right] f^{0}(E), \\
& f^{-}(E)=\frac{1}{2} \exp \left[\left(E+U_{\mathrm{eff}}-E_{F}\right) / k T^{*}\right] f^{0}(E),
\end{aligned}
$$

where $E$ is the $\left(D^{0} \rightarrow D^{+}\right)$transition energy of the dangling bond, $U_{\text {eff }}$ is the effective correlation energy, and $E_{F}$ is the Fermi level. If the hydrogen binding energy $E_{\mathrm{H}}$ is identified with the $D^{0} \rightarrow \mathrm{SiH}$ transition, then the requirement of electronic equilibrium allows us to define the binding energies $E_{\mathrm{H}}^{+}$and $E_{\mathrm{H}}^{-}$, associated with the transitions $D^{+} \rightarrow \mathrm{SiH}$ and $D^{-} \rightarrow \mathrm{SiH}$, respectively, as a function of $E_{F}$ and the electronic levels of the dangling bond ( $E$ and $\left.E+U_{\text {eff }}\right)$ :

$E_{\mathrm{H}}^{+}=E_{\mathrm{H}}-\left(E_{F}-E\right), \quad E_{\mathrm{H}}^{-}=E_{\mathrm{H}}-\left(E+U_{\mathrm{eff}}-E_{F}\right)$.

Thus the grand partition function for $D_{T}$ sites can be defined as

$$
\begin{aligned}
Z=\left\{1+f^{0} \exp \left[-\frac{E_{\mathrm{H}}-\mu_{\mathrm{H}}}{k T^{*}}\right]+f^{+} \exp \left[-\frac{E_{\mathrm{H}}^{+}-\mu_{\mathrm{H}}}{k T^{*}}\right]\right. \\
\left.+f^{-} \exp \left[-\frac{E_{\mathrm{H}}^{-}-\mu_{\mathrm{H}}}{k T^{*}}\right]\right\}^{D_{T}} .
\end{aligned}
$$

This expression can be simplified if we take into account (4)-(6). So, we obtain

$$
Z=\left\{1+2 f^{0} \exp \left[-\left(E_{\mathbf{H}}-\mu_{\mathrm{H}}\right) / k T^{*}\right]\right\}^{D_{T}} .
$$

Then, the average number of hydrogen atoms per site can be calculated:

$$
\mathrm{SiH}=k T^{*} \frac{\delta \ln Z}{\delta \mu_{\mathrm{H}}}=\frac{2 f^{0} \exp \left[-\left(E_{\mathrm{H}}-\mu_{\mathrm{H}}\right) / k T^{*}\right] D_{T}}{1+2 f^{0} \exp \left[-\left(E_{\mathrm{H}}-\mu_{\mathrm{H}}\right) / k T^{*}\right]}
$$

and we can deduce the probability $f_{D}$ of finding a dangling bond at an isolated dangling-bond site, $f_{D}=\left(D_{T}-\mathrm{SiH}\right) / D_{T}$ :

$$
f_{D}=\frac{1}{1+2 f^{0}(E) \exp \left[-\left(E_{\mathrm{H}}-\mu_{\mathrm{H}}\right) / k T^{*}\right]},
$$

where the probability $f_{D}$ of defect formation is expressed in terms of the hydrogen binding energy and the electronic occupation function $f^{0}$. The defect formation energy dependence on Fermi level also implicitly appears in (10). Indeed, the concept of lowering formation energies by charge exchange with the Fermi level may be considered as a consequence of electronic equilibrium between different defect charge states during structural equilibri- um processes.

The use of Eq. (10) to analyze the effects of amorphous spreading of available defect energy levels is straightforward. We only have to apply the statistics to a particular distribution of dangling-bond sites. Thus, if we consider a Gaussian pool of width $\sigma_{P}$, the gap-state distribution can be written as

$$
\begin{aligned}
\frac{d D}{d E}= & \frac{1}{1+f^{0}(E) \exp \left[-\left(E_{\mathrm{H}}-\mu_{\mathrm{H}}\right) / k T^{*}\right]} \\
& \times \frac{\exp \left[-\left(E-E_{P}\right)^{2} / 2 \sigma_{P}^{2}\right]}{\left(2 \pi \sigma_{P}^{2}\right)^{1 / 2}},
\end{aligned}
$$

where $E_{P}$ is the pool position. Note that the possibility of a statistical distribution of $\mathrm{SiH}$ binding energies, $E_{\mathrm{H}}$, and explicit correlations between $E_{\mathrm{H}}$ and the electronic defect energy could be included. We will discuss this below.

From a similar procedure, we now calculate the equilibrium densities of the various hydrogenation states of the $\mathrm{SiSi}$ center. We shall use a simplified description based on reaction (2). We shall consider that the SiSi site can present only two states: unhydrogenated (SiSi) or doubly hydrogenated ( $\mathrm{SiHSiH})$. Then, the SiSi concentration can easily be deduced as

$$
\mathrm{SiSi}=\frac{\mathrm{SiSi}_{\pi}}{1+2 \exp \left[-\left(E_{2 \mathrm{H}}-2 \mu_{\mathrm{H}}\right) / k T^{*}\right.},
$$

where $E_{2 \mathrm{H}}$ is the energy level of hydrogen in the state $\mathrm{SiHSiH}$, namely, the energy of transition $\mathrm{SiSi} \rightarrow \mathrm{SiHSiH}$.

From (11) and (12), superimposing electrical neutrality and a fixed hydrogen chemical potential $\mu_{\mathrm{H}}$ (which could be determined by the deposition conditions ${ }^{10}$ ), both gapstate distribution and hydrogen content $(\sim \mathrm{SiH}+2 \mathrm{SiHSiH})$ can be determined.

\section{B. Nonequilibrium gap-state distribution}

As repeatedly pointed out, ${ }^{1-6}$ the chemical equilibrium theory could also account for general nonequilibrium conditions, such as light soaking, charge injection, and reverse-bias annealing. We now discuss this more general situation, in which the free carrier concentrations will be expressed as a function of the steady-state quasi-Fermi 
levels $E_{F n}$ and $E_{F p}$.

Under nonequilibrium conditions a general function $f^{0}(n, p)$ for the neutral defect state in Eq. (10) may be derived from detailed balance of the various capture and emission processes: ${ }^{14}$

$$
\begin{aligned}
f^{0} & =\frac{1}{1+T^{+}+T^{-}}, \quad T^{+}=\frac{c_{p}^{0} p+e_{n}^{0}}{c_{n}^{+} n+e_{p}^{+}}, \\
T^{-} & =\frac{c_{n}^{0} n+e_{p}^{0}}{c_{p}^{-} p+e_{n}^{-}},
\end{aligned}
$$

where $c_{n}^{0}$ and $c_{p}^{0}$ are the electron- and hole-capture coefficients by $D^{0}, c_{n}^{+}$is the electron-capture coefficient by $D^{+}, c_{p}^{+}$is the hole-capture coefficient by $D^{-}, e_{n}^{0}$ and $e_{p}^{0}$ are the electron and hole emission coefficients from $D^{0}, e_{n}^{-}$is the electron emission coefficient from $D^{-}$, and $e_{p}^{+}$is the hole emission coefficient from $D^{+}$.

However, in order to study whether the density-ofstates (DOS) distribution could be deduced just from Eq. (10) or whether a more general expression is required, we performed a second analysis of the statistical approach. So it is worth emphasizing that the essential hypothesis in the deduction of Eq. (10) consisted in assuming electronic equilibrium between different defect charge states during the chemical equilibrium process. This was taken into account in the formulation of the grand partition function $Z$ both by adding to $E_{\mathrm{H}}$ the binding energies $E_{\mathrm{H}}^{+}$and $E_{\mathrm{H}}^{-}$and by considering the probabilities $f^{0}, f^{+}$, and $f^{-}$. We will now consider a more complex situation in which the energy levels $E_{\mathrm{H}}^{+}$and $E_{\mathrm{H}}^{-}$are split into two new levels $\left(E_{\mathrm{H}}^{+}\right.$into $E_{\mathrm{H} n}^{+}$and $E_{\mathrm{H} p}^{+}$, and $E_{\mathrm{H}}^{-}$into $E_{\mathrm{H} n}^{-}$and $\left.E_{\mathrm{H} p}^{-}\right)$depending on whether the associated charge defect transition $\left(D^{0} \rightarrow D^{+}\right.$and $D^{0} \rightarrow D^{-}$, respectively) involves electronic interchange with the conduction band or with the valence band:

$$
\begin{aligned}
& E_{\mathrm{H} n}^{+}=E_{\mathrm{H}}-\left(E_{F n}-E\right), \\
& E_{\mathrm{H} p}^{+}=E_{\mathrm{H}}-\left(E_{F p}-E\right), \\
& E_{\mathrm{H} n}^{-}=E_{\mathrm{H}}-\left(E+U_{\mathrm{eff}}-E_{F n}\right), \\
& E_{\mathrm{H} p}^{-}=E_{\mathrm{H}}-\left(E+U_{\mathrm{eff}}-E_{F p}\right) .
\end{aligned}
$$

In defining the probabilities for the binding-energy levels in the grand partition function some care is needed. It will now be necessary to take into account the probabilities $f_{n}^{+}$and $f_{p}^{+}$, that a transition $D^{0} \rightarrow D^{+}$involves electron emission or hole capture, and the probabilities $f_{n}^{-}$ and $f_{p}^{-}$, that a transition $D^{0} \rightarrow D^{-}$involves electron capture or hole emission. These probabilities can be deduced from the relations between the rates of electron or hole emission from $D^{0}$ and the rates of electron or hole capture by $D^{0}$ :

$$
\begin{aligned}
& f_{n}^{+}=e_{n}^{0} /\left(c_{p}^{0} p+e_{n}^{0}\right), f_{p}^{+}=\left(c_{p}^{0} p\right) /\left(c_{p}^{0} p+e_{n}^{0}\right), \\
& f_{n}^{-}=\left(c_{n}^{0} n\right) /\left(c_{n}^{0} n+e_{p}^{0}\right), f_{p}^{-}=e_{p}^{0} /\left(c_{n}^{0} n+e_{p}^{0}\right) .
\end{aligned}
$$

In this manner, the hydrogen grand partition function for a number of $D_{T}$ sites, in a nonequilibrium steady-state situation, can be redefined as

$$
\begin{aligned}
Z=\{1 & +f^{0} \exp \left[-\frac{E_{\mathrm{H}}-\mu_{\mathrm{H}}}{k T^{*}}\right]+f^{+} f_{n}^{+} \exp \left[-\frac{E_{\mathrm{H} n}^{+}-\mu_{\mathrm{H}}}{k T^{*}}\right] \\
& +f^{+} f_{p}^{+} \exp \left[-\frac{E_{\mathrm{H} p}^{+}-\mu_{\mathrm{H}}}{k T^{*}}\right] \\
& +f^{-} f_{n}^{-} \exp \left[-\frac{E_{\mathrm{H} n}^{-}-\mu_{\mathrm{H}}}{k T^{*}}\right] \\
& \left.+f^{-} f_{p}^{-} \exp \left[-\frac{E_{\mathrm{H} p}^{-}-\mu_{\mathrm{H}}}{k T^{*}}\right]\right\}^{D_{T}}
\end{aligned}
$$

Using Eqs. (13)-(15) in Eq. (16), simplification of (16) yields just the previous grand partition function (8) (see Appendix). It can thus be concluded that Eq. (11) for the gap-state distribution is valid in both equilibrium and nonequilibrium steady-state conditions.

\section{DISCUSSION}

\section{A. Connection between $E_{\mathrm{H}}$ and $E$}

If the usual approximation of one-electron energies is considered, ${ }^{13}$ the binding energy could be defined as the increase in the electronic energy due to occupation of a neutral defect state by one hydrogen: $E_{\mathrm{H}}=E(\mathrm{SiH})-E$, where $E(\mathrm{SiH})$ is the monoelectronic energy associated with the SiH bond. Starting from this definition, two alternative descriptions could be considered. First, we have performed a calculation of the DOS distribution taking $E(\mathrm{SiH})$ as a constant (model $\mathrm{A}$ in Fig. 1). Note that this hypothesis has been implicitly assumed in the conventional defect pool approach. With this approximation, and ignoring the cutoff of $D(E)$ towards lower energies as a result of complete depletion of available defect sites, the DOS distribution obtained can then be split into three defect bands, $D^{0}, D^{+}$, and $D^{-}$, where the $D^{+}$ band is centered at $E_{P}$ and the $D^{-}$and $D^{0}$ bands are shifted by $-2 \sigma_{P}^{2} / k T^{*}$ and $-\sigma_{P}^{2} / k T^{*}$ from the $D^{+}$position (see Fig. 2). This result is similar to that obtained for the conventional defect pool model ${ }^{4-6}$ except for the

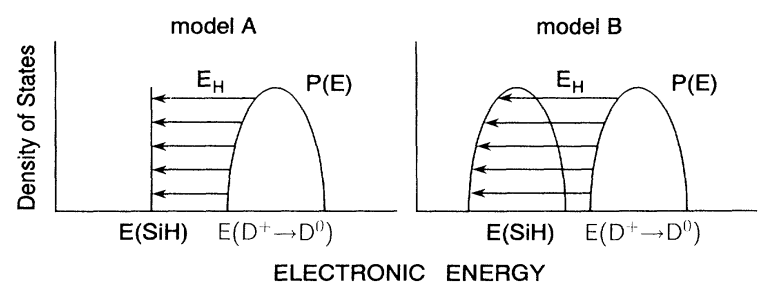

FIG. 1. Schematic density-of-states diagram, to illustrate the two extreme descriptions of the hydrogen binding energy $E_{H}$ according to the approximation of one-electron energies: $E_{\mathrm{H}}=E(\mathrm{SiH})-E\left(D^{+} \rightarrow D^{0}\right)$. In model $\mathrm{A}$, the monoelectronic energy associated with the $\mathrm{SiH}$ bond is taken to be a constant. Thus $E_{\mathrm{H}}$ is a function of the corresponding electronic defect energy: $E_{\mathrm{H}}=$ const $-E$. In model $\mathrm{B}$, the binding energy $E_{\mathrm{H}}$ is independent of the electronic defect energy. It is assumed $E_{\mathrm{H}}=$ const. 


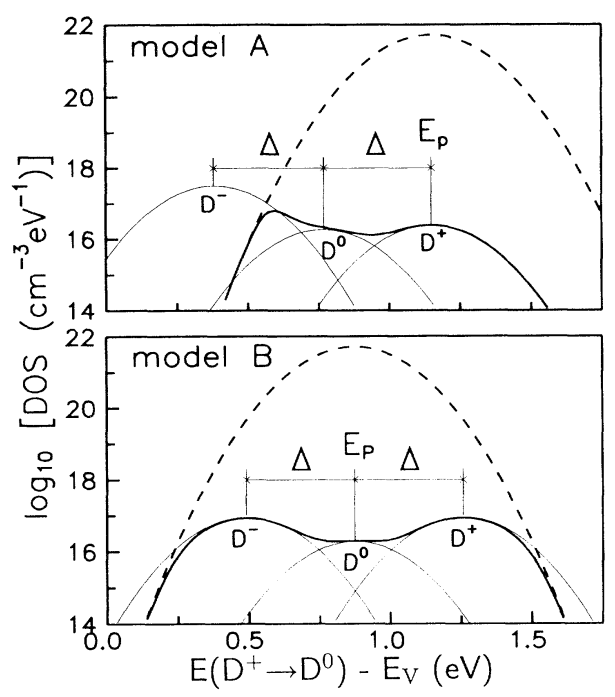

FIG. 2. Equilibrium gap-state defect distributions for model A $\left(E_{\mathrm{H}}=\right.$ const $\left.-E\right)$ and model B $\left(E_{\mathrm{H}}=\right.$ const $)$. Thin solid line represents its deconvolution in the three defect bands, $D^{+}, D^{0}$, and $D^{-}$, when pool depletion is ignored. The band shift $\Delta$ is $\sigma_{P}^{2} / k T^{*}$ for both models. Dashed line represents the Gaussian defect pool $P(E)$. Note that different pool positions were assumed for each approximation (see text).

term $k T^{*}$ which, in the conventional treatment, is replaced by the width of the exponential weak-bond distribution $k T_{V}$.

On the other hand, we have considered a second situation in which the binding energy $E_{\mathrm{H}}$ is independent of the electronic energy $E$ of the defect created, in other words, when the possible random fluctuation of $E_{\mathrm{H}}$ is not correlated with the pool distribution (model B in Fig. 1). In this case, it can be shown that the $D^{0}$ band is formed at the pool center $E_{P}$ and the $D^{-}$and $D^{+}$bands are shifted by $-\sigma_{P}^{2} / k T^{*}$ and $+\sigma_{P}^{2} / k T^{*}$ from the $D^{0}$ position (see Fig. 2).

\section{B. Dark equilibrium DOS}

If the hydrogen chemical potential is fixed, a selfconsistent implicit numerical solution for the equilibrium density of states $D(E)$ and the Fermi level $E_{F}$ can be obtained from Eq. (11) by the requirement of charge neutrality:

$$
\begin{aligned}
& Q\left(E_{f}, \mu_{\mathrm{H}}\right)=p-n+Q_{\mathrm{BT}}\left(E_{F}\right) \\
&+\int D\left(E, E_{F}, \mu_{\mathrm{H}}\right) \\
& \quad \times\left[f^{+}\left(E, E_{F}\right)-f^{-}\left(E, E_{F}\right)\right] d E=0,
\end{aligned}
$$

where $Q_{\mathrm{BT}}$ represents the trapped carrier concentration in the band tail states.

We take the experimentally determined values for the parameters summarized in Table $I$ as constants corresponding to standard electronic grade amorphous silicon. ${ }^{15}$ The values of the pool position $E_{P}$ and the hydrogen chemical potential $\mu_{\mathrm{H}}$ have been determined imposing a Fermi level lying $0.8 \mathrm{eV}$ below the conduction band and a neutral defect density in the range of $10^{15} \mathrm{~cm}^{-3}$ for undoped material in neutral conditions. So, from Fig. 2, different pool positions were assumed for each approximation (model A or B) in order to make the electronic properties deduced from the gap-state distribution agree with experimental results.

With the preceding parameter settings, only two free parameters remain to be investigated both for models A and $\mathbf{B}$. These parameters are the defect pool width $\sigma_{P}$ and the energy difference between the clustered hydrogen and isolated hydrogen $\delta E$ defined as $E_{2 \mathrm{H}} / 2-E_{\mathrm{H}} \cdot \delta E$

TABLE I. Parameters used in the calculations.

\begin{tabular}{llc}
\hline \hline Parameter & \multicolumn{1}{c}{ Description } & \multicolumn{1}{c}{ Value } \\
\hline$E_{C}-E_{V}$ & Band gap & $1.75 \mathrm{eV}$ \\
$N_{C}, N_{V}$ & Effective densities of state & $2 \times 10^{20} \mathrm{~cm}^{-3}$ \\
$T_{V}$ & Valence-band temperature & $550 \mathrm{~K}$ \\
$T_{C}$ & Conduction-band temperature & $400 \mathrm{~K}$ \\
$N_{\mathrm{VBT}}$ & Density of valence-band tail states in $E_{V}$ & $1 \times 10^{21} \mathrm{~cm}^{-3} \mathrm{eV}^{-1}$ \\
$N_{\mathrm{CBT}}$ & Density of conduction-band tail states in $E_{C}$ & $1 \times 10^{21} \mathrm{~cm}^{-3} \mathrm{eV}^{-1}$ \\
$\sigma_{\mathrm{BT}}$ & Charged band tail capture cross section & $10^{-15} \mathrm{~cm}^{2}$ \\
$\sigma_{\mathrm{BT}}^{0}$ & Neutral band tail capture cross section & $10^{-17} \mathrm{~cm}^{2}$ \\
& & \\
$U_{\mathrm{eff}}$ & Effective correlation energy & $0.2 \mathrm{eV}^{0}$ \\
$\sigma_{n}^{0}$ & $D^{0}$ electron-capture cross section & $2.7 \times 10^{-15} \mathrm{~cm}^{2}$ \\
$\sigma_{p}^{0}$ & $D^{0}$ hole-capture cross section & $8 \times 10^{-15} \mathrm{~cm}^{2}$ \\
$\sigma_{n}^{+}$ & $D^{+}$electron-capture cross section & $1.3 \times 10^{-14} \mathrm{~cm}^{2}$ \\
$\sigma_{p}^{-}$ & $D^{-}$hole-capture cross section & $2 \times 10^{-14} \mathrm{~cm}^{2}$ \\
$\mathrm{SiH}_{T}$ & & \\
$\mathrm{SiSi}_{T}$ & Density of SiH sites & $1.5 \times 10^{21} \mathrm{~cm}^{-3}$ \\
\hline \hline
\end{tabular}


determines the thermal activation energy of $D_{0}$ at equilibrium in the hydrogen-deficit model of Zafar and Schiff, ${ }^{8}$ where the activated behavior of the spin density is interpreted as a gradual transfer of bonded hydrogen from the dilute phase to the clustered phase as the temperature rises. However, the incorporation of the defect pool and the dependence of the defect formation energy on the Fermi level could modify this interpretation, as we shall discuss in the next paragraph.

Temperature dependence of $D^{0}$. It could be demonstrated that above the equilibration temperature, models $\mathrm{A}$ and $\mathrm{B}$ both predict a thermally activated spin density. Figure 3 shows the activation energy $E_{\text {act }}$ as a function of $\delta E$ for both models, using different values of $\sigma_{P}$. Two behavior patterns can be deduced for the dependence of $E_{\text {act }}$ on $\delta E$. For high values of $\delta E$, that is, when hydrogen is more weakly bound to the clustered phase, there is a linear dependence of $E_{\text {act }}$ on $\delta E$. In these conditions, the change of $D_{0}$ with temperature has the same interpretation as in the model of Zafar and Schiff. However, the binding-energy difference $\delta E$ can only be equated to the thermal activation energy for model B. Model A predicts a smaller value of $E_{\text {act }}$ than $\delta E$, depending on the pool width $\sigma_{P}$ (with small values of $\sigma_{P}, \delta E$ becomes equal to $E_{\text {act }}$ ). On the other hand, for low values of $\delta E$, that is, when the binding energies for the clustered and isolated hydrogen are similar, $E_{\text {act }}$ is independent of $\delta E$. In this regime, the change of $D_{0}$ with temperature can be explained as a consequence of hydrogen redistribution in the isolated sites that involves a change in the state of charge of the defects. Note that, independently of the value of $\sigma_{P}$, for model $\mathrm{A}$ the activated behavior of the spin density is essentially lost in this latter regime, whereas for model B a clear dependence of $E_{\text {act }}$ on $\sigma_{P}$ is observed.

Experimentally, the spin density in $a-\mathrm{Si}: \mathrm{H}$ above $200^{\circ} \mathrm{C}$

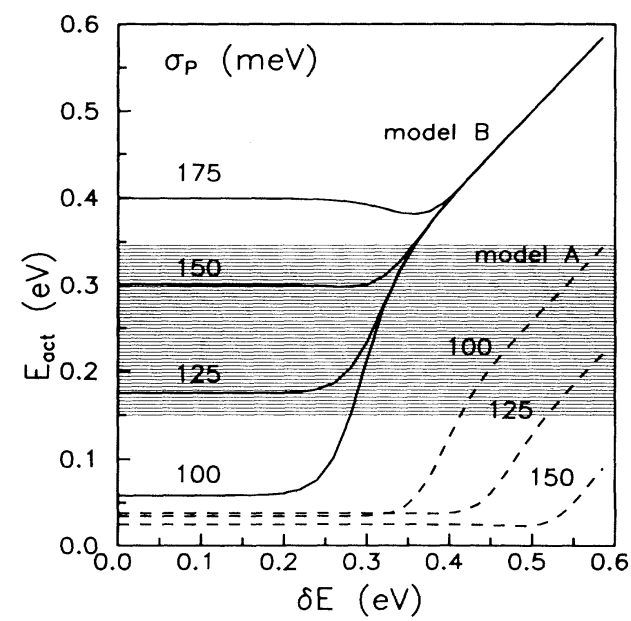

FIG. 3. Activation energy of the spin density at $T=200^{\circ} \mathrm{C}$ as a function of the hydrogen binding-energy difference $\delta E=E_{2 \mathrm{H}} / 2-E_{\mathrm{H}}$. Several defect pool widths are considered for both model A (dashed line) and B (solid line). The shaded region represents the range of reported values for the activation energy (Refs. 1, 15, and 16). has an activation energy of order $0.3 \mathrm{eV} .{ }^{1}$ Other reported values are between 0.15 and $0.35 \mathrm{eV} .{ }^{15,16}$ This range of activation energies can only be obtained with both models $A$ and $B$ for a suitably high value of $\delta E$, and only with model B for lower values of $\delta E$ if the pool width lies in the 120-170-meV range. Note that these values of the pool width are in agreement with those usually suggested for the defect pool. ${ }^{3-5}$ The viability of a description based on model $\mathrm{B}$, with a low $\delta E$ and a pool width lying in the range $120-170 \mathrm{meV}$, will be reinforced when we consider the nonequilibrium situation. In fact, it will be shown in Sec. III $\mathrm{C}$ that the chemical model based on the two phases of bound hydrogen can only explain lightinduced creation of defects if stable enough clustered hydrogen is assumed.

Gap-state dependence of Fermi level. The concept of the defect pool and the fact that the energy cost of forming a defect at any particular site can depend on the charge state, together imply that the characteristic properties of defects in $a$-Si:H will depend on the position of the Fermi level during equilibration. As has been pointed out $^{4}$ these effects can explain the apparent experimental contradiction between electron-spin resonance data, which require a positive correlation energy, and equilibrium gap-state spectra, which show $D^{-}$in $n$-type $a$-Si:H to lie deeper than $D^{0}$ in undoped $a-\mathrm{Si}: \mathrm{H}$.

The dependence of $D(E)$ on Fermi level explicitly appears in Eq. (11) through $f^{0}(E)$. The effect of varying the Fermi level when a constant $\mu_{\mathrm{H}}$ is assumed is shown in Fig. 4 (model $\mathrm{B}$ is considered for $\sigma_{P}=150 \mathrm{meV}$ and $\delta E=0)$. It can be seen that as material becomes $n$ type the $D^{-}$band is enhanced in comparison with the $D^{+}$, and vice versa as material becomes $p$ type. The use of hydrogen chemical potential $\mu_{\mathrm{H}}$ allows a more realistic interpretation of the effects induced by Fermi-level displacement. For example, in calculating the spatially vari-

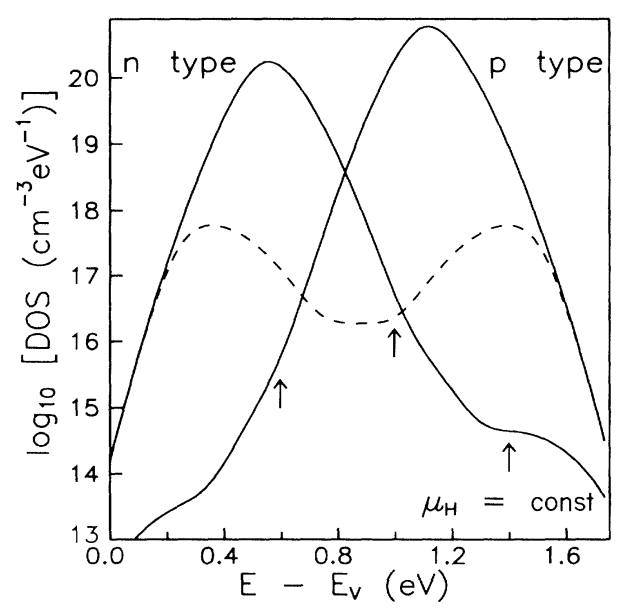

FIG. 4. Equilibrium gap-state defect distributions for intrinsic (dashed line), $n$-type and $p$-type $a$-Si:H calculated using Eq. (11). Arrows indicate the Fermi-level position. Model B is considered, with $\sigma_{P}=150 \mathrm{meV}$ and $E_{P}-E_{V}=0.875 \mathrm{eV}$. The hydrogen chemical potential is the same in each case, $\mu_{\mathrm{H}}-E_{\mathrm{H}}=0.5 \mathrm{eV}$. 
ant defect density in the $i$ layer in $p-i-n$ solar cell structures, where it is expected that the dependence of $D(E)$ on $E_{F}$ leads to a high density of defect states near the interfaces, it is clear that $\mu_{\mathrm{H}}$ must remain constant along the device when hydrogen chemical equilibrium is assumed. Equation (11) is also compatible with the recent $a-\mathrm{Si}: \mathrm{H}$ growth model of Street, ${ }^{9,10}$ which suggests that, at sufficiently high growth temperature, $\mu_{\mathbf{H}}$ in the plasma and the film tend to equalize because of the rapid exchange of hydrogen across the growth surface.

Defect density in hydrogen-depleted a-Si:H. As mentioned in the Introduction, one of the attractive features of the model of Zafar and Schiff based on reaction (2) is that it can predict the changes in spin density which occur when hydrogen is removed from the material or replaced. In particular, if a high enough value of binding energy $\delta E$ is assumed, this model can account for the square-root dependence of the defect concentration on the depleted hydrogen concentration upon thermal annealing of $a-\mathrm{Si}: \mathrm{H}$. As can be seen in Fig. 5, where the spin density is shown as a function of the percentage of hydrogen release (for model $\mathrm{B}$, and assuming $\sigma_{P}=150$ $\mathrm{meV}$ ), the incorporation of the defect pool concept and the dependence of the defect formation energies on the Fermi level does not significantly modify this result. For $\delta E \approx 0.3 \mathrm{eV}$, we obtain the same sublinear dependence of the spin density upon hydrogen deficit, but for lower values of $\delta E$ this result is not found. However, as shall be discussed in the next section, light-induced creation of defects can only be explained if a stable enough clustered hydrogen is assumed $(\delta E \approx 0 \mathrm{eV})$. Nevertheless, in our opinion, the interpretation of the hydrogen evolution experiments should be carefully reconsidered. Although it is reasonable to assume that hydrogen effusion proceeds from the hydrogen clustered phase, this effusion is produced in experimental conditions which are different

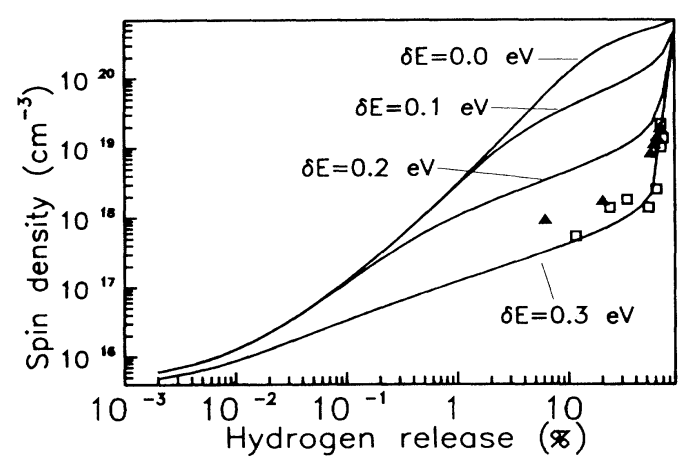

FIG. 5. Calculated dependence of the spin density upon the percentage of hydrogen release for several values of the hydrogen binding-energy difference $\delta E=E_{2 \mathrm{H}} / 2-E_{\mathrm{H}}$. The same model parameters as in Fig. 4 are considered. In each case, the initial value of $\mu_{\mathrm{H}}$ has been calculated in order to make $D^{0}=1 \times 10^{15} \mathrm{~cm}^{-3}$ at $300 \mathrm{~K}$. Symbols indicate the measured points based on spin density and deuterium profiles in samples which were first depleted of hydrogen and then plasma deuterated: triangles indicate $350^{\circ} \mathrm{C}$ deuteration and squares $400^{\circ} \mathrm{C}$ deuteration (from Ref. 24). from those of the other defect phenomena studied here. So, in analyzing activated behavior of the spin density, Fermi-level displacement, or metastable effects, one of the basic hypotheses assumes that $a-\mathrm{Si}: \mathrm{H}$ structural properties (defect pool parameters, total density of SiSi sites) do not vary significantly in such processes. However, this hypothesis may not be valid in hydrogen effusion processes, where there is experimental evidence of an important lattice reconstruction upon hydrogen removal.

\section{Steady-state light-induced defect density in $a$-Si:H}

So far we have been concerned only with electronic equilibrium. However, we have seen in Sec. II B that expression (11) for the gap-state distribution $D(E)$ may be valid for more general nonequilibrium steady-state conditions. In fact, we have only to consider the general electronic occupation function $f^{0}(n, p)$, derived from a detailed balance of the various capture and emission processes [Eq. (13)], in Eq. (11).

In what follows, we explore the consequences that different hypotheses about hydrogen binding energies and pool parameters have in the gap-state distribution for light-soaked undoped $a-\mathrm{Si}: \mathrm{H}$. As in Sec. III B we adjust the values of the pool position $E_{P}$ and the equilibrium hydrogen chemical potential $\mu_{\mathrm{H} 0}$ in order to give the measured spin density in the annealed state (at room temperature) and the Fermi level lying at $E_{C}-0.8 \mathrm{eV}$. If the hydrogen concentration of the sample remains constant, we expect that reequilibration induced by illumination alters the value of the hydrogen chemical potential $\mu_{\mathrm{H}} \neq \mu_{\mathrm{HO}}$. Thus $D(E)$ can be considered as a function of $\mu_{\mathrm{H}}$, and the steady-state quasi-Fermi levels $E_{F n}$ and $E_{F p}$. The lightsoaked $D(E)$ is calculated by solving the following system of equations for $\mu_{\mathrm{H}}, E_{F p}$, and $E_{F n}$ :

$$
\begin{aligned}
& Q\left(\mu_{\mathrm{H}}, E_{F p}, E_{F n}\right)=0, \\
& R\left(\mu_{\mathrm{H}}, E_{F p}, E_{F n}\right)=G, \\
& H\left(\mu_{\mathrm{H}}, E_{F p}, E_{F n}\right)=H_{0},
\end{aligned}
$$

where Eq. (18a) represents the charge neutrality condition, Eq. (18b) represents the requirement that, in steady-state conditions, generation rate $G$ must equalize recombination rate $R$, and Eq. (18c) represents the condition of hydrogen content constant. The theory of Simmons and Taylor ${ }^{17}$ for arbitrary distributions of trapping levels and the statistics treated by Sah and Shockley ${ }^{18}$ for multiple energy-level defect states are used to calculate the net trapped charge and the rate of recombination in the band tails and dangling bonds. Thus the set of coupled equations (18) is linearized and solved using the method of Newton. Once the light-soaked $D(E)$ has been calculated, statistics under dark equilibrium conditions is applied to determine the spin density.

Intensity and temperature dependence of light-induced defect density. The temperature dependence of the spin density in the annealed state before soaking (solid lines) and in the saturated light-soaked state (dashed lines) for model $B$ (that is, assuming $E_{H}$ to be constant) are presented in Figs. 6 and 7 for several values of $\delta E$ and 


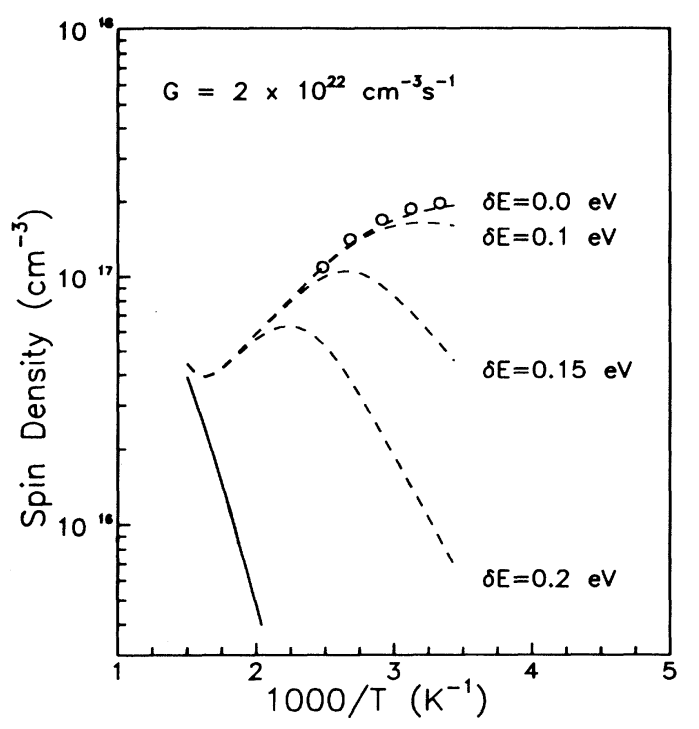

FIG. 6. Calculated spin density in the annealed state before illumination (solid line) and in the saturated state when a carrier generation rate of $2 \times 10^{22} \mathrm{~cm}^{-3} \mathrm{~s}^{-1}$ is assumed (dashed line), for model B (with $\sigma_{P}=165 \mathrm{meV}$ ) and several values of $\delta E$. Circles indicate the experimental data for the saturated spin density reported by Hata, Isomura, and Wagner (Refs. 19 and 20). Note that the temperature dependence of the defect density in the annealed state is independent of $\delta E$.

$\sigma_{P}$. The open circles in both figures correspond to the measured value of saturated defect density reported by Hata, Isomura, and Wagner. ${ }^{19,20}$ Making $\sigma_{P}=0.165 \mathrm{eV}$ and $\delta E=0$ [and adjusting $\mu_{\mathrm{H}}$ to obtain an annealed neutral defect density of $2 \times 10^{15} \mathrm{~cm}^{-3}$ (Ref. 20)], the form and magnitude of the measurements are well reproduced by the model. It can be observed in Fig. 6 that the calculated results for high values of $\delta E$ do not permit an ex-

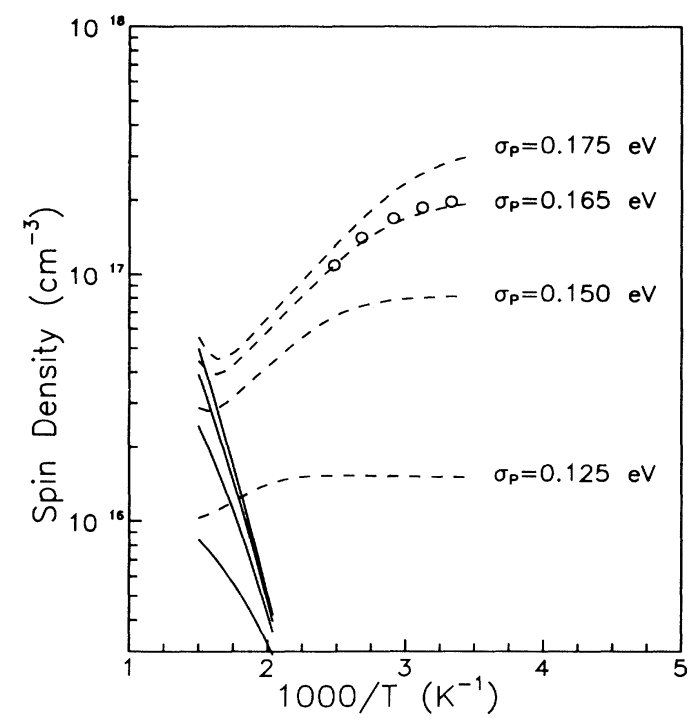

FIG. 7. Calculated temperature dependence of the spin density in the annealed state (solid line) and in the saturated state (dashed line, $G=2 \times 10^{22} \mathrm{~cm}^{-3} \mathrm{~s}^{-1}$ ) as a function of the pool width $\sigma_{P}$. Model B with $\delta E=0 \mathrm{eV}$ is assumed. Circles indicate the experimental data of Fig. 6. planation of the Staebler-Wronski effect. If hydrogen is weakly bound to the clustered phase, new statistical requirements imposed by illumination mainly involve the transfer of bonded hydrogen from the clustered phase to the dilute phase so the creation of new neutral defects is nearly inhibited. In contrast, if a small value of $\delta E$ is considered $(\delta E \sim 0)$ hydrogen redistribution in the isolated phase leads to a considerable increase in spin density, in accordance with experimental results. These conclusions are also valid for model $\mathrm{A}$ (that is, assuming $E_{\mathrm{H}}=$ const $-E$ ). However, as we saw in Sec. III B, for model $\mathrm{A}$ the activated behavior of the dark spin density is essentially lost when a small $\delta E$ is considered.

The dependence of the density of light-induced neutral defects on the illumination intensity and temperature, also for model B and assuming $\delta E=0$, is shown in Fig. 8 . It can be observed that the calculated results agree well with experimental data reported by Hata, Isomura, and Wagner. ${ }^{19,20}$ The saturation spin density decreases with increasing temperature, and the effect is stronger for low illumination intensities. Note that at room temperature the spin density is nearly independent of the illumination intensity. For higher temperatures a power-law dependence is found in the low illumination range. The same behavior has also been reported by Santos, Jackson, and Street. ${ }^{21}$ However, the magnitude of the saturated defect density found cannot be reproduced with the same pool width $\sigma_{P}$ and hydrogen chemical potential $\mu_{\mathrm{H}}$ as those used to fit the experimental results of Hata, Isomura, and Wagner.

We emphasize that the introduction of the defect pool concept is essential for interpreting the Staebler-Wronski effect in this chemical equilibrium framework. The ratio of charged to neutral defect densities sensitively depends on the pool width $\sigma_{P}$. If a wide enough pool is assumed $\left(\sigma_{P} \sim 0.15 \mathrm{eV}\right)$ the two charged and shallow defect bands are significantly higher than the neutral defect band in dark equilibrium [dashed line in Fig. 9(a)]. Hydrogen redistribution induced by illumination involves the "conversion" of these charged and shallow centers to neutrals near the middle of the gap [solid line in Fig. 9(a)].

Amorphous silicon prepared under visible-light illumination. As we suggest in the Introduction, explicit

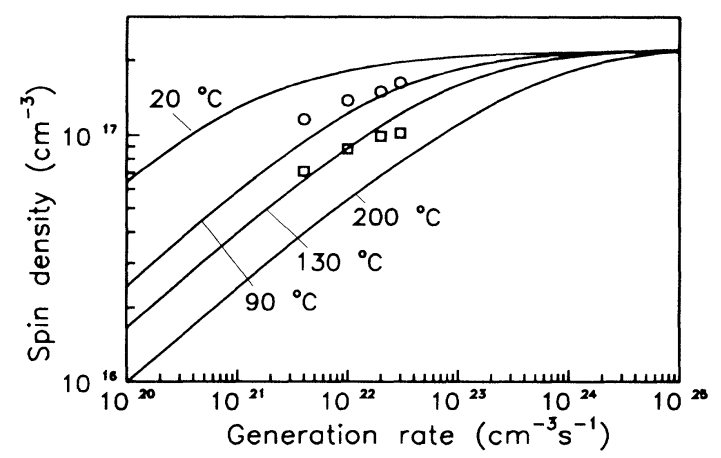

FIG. 8. Calculated steady-state spin densities as function of carrier generation rate for several temperatures. Model $\mathrm{B}$ is considered with $\sigma_{P}=165 \mathrm{meV}$ and $\delta E=0 \mathrm{eV}$. Symbols indicate the experimental data reported by Hata, Isomura, and Wagner (Refs. 19 and 20): circles for $90^{\circ} \mathrm{C}$ and squares for $130^{\circ} \mathrm{C}$. 


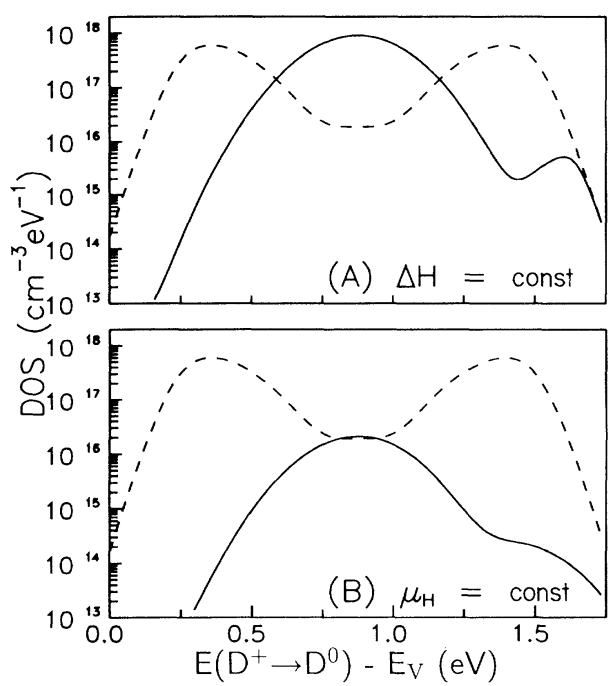

FIG. 9. Saturated light-soaked defect DOS (solid line) under high illumination conditions $\left(G=4 \times 10^{22} \mathrm{~cm}^{-3} \mathrm{~s}^{-1}\right)$ and equilibrium defect DOS (dashed line) from two different viewpoints: (A) $a-\mathrm{Si}: \mathrm{H}$ is considered as a closed system for hydrogen, that is, the hydrogen content $\Delta \mathrm{H}$ remains constant. (B) $a-\mathrm{Si}: \mathrm{H}$ is considered as an open system for hydrogen, as one can expect for growing conditions. In these conditions, it is assumed that the hydrogen chemical potential $\mu_{\mathrm{H}}$ remains constant and equal to $\mu_{\mathrm{H}}$ in the plasma.

use of hydrogen chemical potential allows a realistic description of complex situations. We refer here to visible light illumination during $a-\mathrm{Si}: \mathbf{H}$ growth. In these conditions, the film should be assumed as an open system for hydrogen. So, in accordance with the growth model of Street, ${ }^{9,10} \mu_{\mathrm{H}}$ should remain invariable and equal to the hydrogen chemical potential in the plasma. Thus we have reworked the previous calculation for a constant $\mu_{\mathrm{H}}$ $\left(\mu_{\mathrm{H}}=\mu_{\mathrm{H} 0}\right)$. The result is shown in Fig. 9(b). The $D^{+}$and $D^{-}$sites are saturated, but now the $D^{0}$ band is only slightly higher than in the dark equilibrium state. This result is in agreement with some experimental data, ${ }^{22,23}$ which show that visible-light illumination during growth can result in both smaller light-induced changes and good electronic properties.

\section{CONCLUSIONS}

We have presented a model of the gap-state distribution of $a-\mathrm{Si}: \mathrm{H}$ which is based on both phases of bonded hydrogen observed by NMR. In fact, the model is an extension of the hydrogen-statistical description of Zafar and Schiff, ${ }^{8}$ which includes the defect pool concept and the dependence of formation energies of the Fermi level. It is demonstrated that the expression obtained for the DOS is valid for both equilibrium and nonequilibrium electronic conditions. The wide range of hypotheses that can be tested with our statistical description could have important consequences in a new interpretation of experimental results. So, we found that a model in which the hydrogen binding energy is independent of the monoelectronic defect energy, if a wide enough pool is assumed, allows a unified description of different defect phenomena in $a-\mathrm{Si}: \mathrm{H}$ : (i) The thermally activated paramagnetic defect density, (ii) the dependence of the gap-state distribution on the Fermi-level position, and (iii) the intensity and temperature dependence of the steady-state light-induced defect density. This unified description is only possible if the hydrogen binding energies for the clustered and isolated phases are similar.

\section{ACKNOWLEDGMENT}

This work has been supported by the DGICYT of the Spanish Government (Project No. PB89-0236).

\section{APPENDIX}

In this appendix we will demonstrate that the simplification of the general grand partition function (16) yields (8). First, we will express the general occupation probabilities in terms of $f^{0}$ :

$$
f^{+}=T^{+} f^{0}, f^{-}=T^{-} f^{0},
$$

where $T^{+}$and $T^{-}$are defined in (13). The grand partition function (16) may now be rewritten in the form

$$
\begin{aligned}
Z=\left\{1+f^{0} \exp \left[-\frac{E_{\mathrm{H}}-\mu_{\mathrm{H}}}{k T^{*}}\right]\right. & {\left[T^{+} f_{n}^{+} \exp \left[\frac{E_{F n}-E}{k T^{*}}\right]+T^{+} f_{p}^{+} \exp \left[\frac{E_{F p}-E}{k T^{*}}\right]\right.} \\
& \left.\left.+T^{-} f_{n}^{-} \exp \left[\frac{E+U_{\mathrm{eff}}-E_{F n}}{k T^{*}}\right]+T^{-} f_{p}^{-} \exp \left[\frac{E+U_{\mathrm{eff}}-E_{F p}}{k T^{*}}\right]\right]\right\}^{D_{T}} .
\end{aligned}
$$

Considering the relationships between the capture and emission coefficients, the exponential in the parentheses in (A2) could be expressed in terms of these coefficients:

$$
\begin{aligned}
& \exp \left[\left(E_{F n}-E\right) / k T^{*}\right]=\frac{1}{2} c_{n}^{+} n / e_{n}^{0}, \\
& \exp \left[\left(E_{F p}-E\right) / k T^{*}\right]=\frac{1}{2} e_{p}^{+} /\left(c_{p}^{0} p\right), \\
& \exp \left[\left(E+U_{\mathrm{eff}}-E_{F n}\right) / k T^{*}\right]=\frac{1}{2} e_{n}^{-} /\left(c_{n}^{0} n\right), \\
& \exp \left[\left(E+U_{\mathrm{eff}}-E_{F p}\right) / k T^{*}\right]=\frac{1}{2} c_{p}^{-} p / e_{p}^{0} .
\end{aligned}
$$

On the other hand, we can introduce the following relationships which are deduced from (13) and (15):

$$
\begin{aligned}
& T^{+} f_{n}^{+}=e_{n}^{0} /\left(c_{n}^{+} n+e_{p}^{+}\right), \quad T^{+} f_{p}^{+}=c_{p}^{0} p /\left(c_{n}^{+} n+e_{p}^{+}\right), \\
& T^{-} f_{n}^{-}=c_{n}^{0} n /\left(c_{p}^{-}+e_{n}^{-}\right), \quad T^{-} f_{p}^{-}=e_{p}^{0} /\left(c_{p}^{-} p+e_{n}^{-}\right) .
\end{aligned}
$$

Then, inserting (A3) and (A4) in (A2) and making straightforward algebraic transformations, we again find the grand partition (8). 
1Z. E. Smith and S. Wagner, in Advances in Amorphous Semiconductors, edited by H. Fritzsche (World Scientific, Singapore, 1989), p. 409.

${ }^{2}$ Y. Bar-Yam, D. Adler, and J. D. Joannopoulos, Phys. Rev. Lett. 57, 467 (1986).

${ }^{3}$ K. Winer, Phys. Rev. Lett. 63, 1487 (1989).

${ }^{4}$ K. Winer, Phys. Rev. B 41, 12150 (1990).

${ }^{5}$ G. Schumm and G. H. Bauer, J. Non-Cryst. Solids 137/138, 315 (1991).

${ }^{6}$ R. A. Street and K. Winer, Phys. Rev. B 40, 6236 (1989).

${ }^{7}$ S. Zafar and E. A. Schiff, in Amorphous Silicon Technology1990, edited by P. C. Taylor, M. J. Thompson, P. G. LeComber, Y. Hamakawa, and A. Madan, MRS Symposia Proceedings No. 192 (Materials Research Society, Pittsburgh, 1990), p. 139.

${ }^{8}$ S. Zafar and E. A. Schiff, Phys. Rev. B 40, 5235 (1989).

${ }^{9}$ R. A. Street, J. Non-Cryst. Solids 137/138, 645 (1991).

${ }^{10}$ R. A. Street, Phys. Rev. B 43, 2454 (1991).

${ }^{11}$ J. Baum, K. K. Gleason, A. Pines, A. N. Garroway, and J. A. Reimer, Phys. Rev. Lett. 56, 1377 (1986).

${ }^{12}$ D. Adler and E. J. Yoffa, Phys. Rev. Lett. 36, 1197 (1976).

${ }^{13}$ M. Stutzmann, Philos. Mag. B 56, 63 (1987).
${ }^{14}$ W. Shockley and J. T. Last, Phys. Rev. 107, 392 (1957).

${ }^{15}$ R. A. Street, in Hydrogenated Amorphous Silicon, edited by R. W. Cahn, E. A. Davis, and I. M. Ward (Cambridge University Press, Cambridge, England, 1991).

16T. J. McMahon, in Amorphous Silicon Technology-1991, edited by A. Madan, Y. Hamakawa, M. Thompson, P. C. Taylor, and P. G. LeComber, MRS Symposia Proceedings No. 219 (Materials Research Society, Pittsburgh, 1991), p. 57.

17J. G. Simmons and G. W. Taylor, Phys. Rev. B 4, 502 (1971).

${ }^{18}$ C. T. Sah and W. Shockley, Phys. Rev. 109, 1103 (1958).

${ }^{19}$ N. Hata, M. Isomura, and S. Wagner, Appl. Phys. Lett. 60, 1462 (1992).

${ }^{20}$ M. Isomura, N. Hata, and S. Wagner, J. Non-Cryst. Solids 137, 223 (1991).

${ }^{21}$ P. V. Santos, W. B. Jackson, and R. A. Street, Phys. Rev. B 44, 12800 (1991).

${ }^{22}$ I. Sakata, M. Yamanaka, and Y. Hayashi, J. Appl. Phys. 67, 3737 (1990).

${ }^{23}$ I. Sakata, M. Yamanaka, and Y. Hayashi, Jpn. J. Appl. Phys. 30, L326 (1991).

${ }^{24}$ W. B. Jackson, C. C. Tsai, and R. Thompson, Phys. Rev. Lett. 64, 56 (1990). 